\title{
Former Hormonal Contraceptive User
}

National Cancer Institute

\section{Source}

National Cancer Institute. Former Hormonal Contraceptive User. NCI Thesaurus. Code C160672.

The individual is a former user of hormonal contraceptives. 